\title{
Perforated Duodenal Ulcer in a Young Child: An Uncommon Condition
}

\author{
Yadav RP, ${ }^{1}$ Agrawal CS, ' Gupta RK,' Rajbansi S, ${ }^{1}$ Bajracharya A, ${ }^{1}$ Adhikary S' \\ 'Department of Surgery, BP Koirala Institute of Health Sciences, Dharan, Nepal
}

\begin{abstract}
Duodenal ulcer is an uncommonly diagnosed entity in children. H. pylori infection, blood group ' $\mathrm{O}^{\prime}$ or secondary to medications like non steroidal anti-inflammatory drugs (NSAID) and corticosteroids or physiological stress in burns, head injury and mucosal ischemia are implicated as risk factors for their causation. The diagnosis is usually overlooked because of vague and variable symptoms and remote index of suspicion accounted for their low incidence in children. Undiagnosed or mistreated perforations may carry high morbidity and mortality. We report a successfully treated $41 / 2$ year old male child who presented with features of perforation peritonitis and was incidentally found to have a perforated duodenal ulcer.
\end{abstract}

Key Words: duodenal ulcer, laparotomy, perforation

\section{INTRODUCTION}

Duodenal ulcer is an uncommonly diagnosed entity in children with an incidence of 1.55 cases per year. ${ }^{1}$ Because of its rarity, its diagnosis is usually overlooked unless it presents with complications. The usual manifestations of a complicated duodenal ulcer are perforation and hemorrhage. Even in such cases which present as acute abdomen, it is not considered as a possible diagnosis because of low index of suspicion.

\section{CASE REPORT}

A four and half year male child presented to the surgical emergency with 4 days history of pain abdomen, bilious vomiting and abdominal distention. There was no past history suggestive of peptic ulcer disease in him or in the family. The child was apparently healthy, was not on any medication before the onset of the illness. History revealed that he was born at full term at home with no significant prenatal or post natal complications. There was no previous history of hospitalization or a medical problem and immunization was up-to date.

On physical examination, the child was lethargic and dehydrated. He had tachycardia but no tachypnea or fever. Cardiac examination was within normal limits. The abdomen was distended, tender and demonstrated guarding with rebound tenderness. Liver dullness was masked and bowel sounds were absent. Rectal examination revealed no significant findings. An upright abdominal radiograph with both domes of the diaphragm revealed presence of pneumoperitoneum (Figure 1).

\footnotetext{
Correspondence:

Dr. Rohit Prasad Yadav

Department of Surgery

BP Koirala Institute of Health Sciences

Dharan, Nepal.

Email: yadavrohit|@yahoo.com

Phone: 9842023269
} 
Routine laboratory investigations were unremarkable. $\mathrm{He}$ had hemoglobin of $11.7 \mathrm{gm} / \mathrm{dl}$ and ' $O$ ' positive blood group. The patient was taken up for exploratory laparotomy after resuscitation with a provisional diagnosis of hollow viscus perforation peritonitis. At surgery, a small perforation was seen in the anterior wall of the first part of duodenum (Figure 2) with bilious collection. There was moderate contamination of the peritoneal cavity. Simple closure with omental patch followed by peritoneal lavage was done and the abdomen closed with drain in the subhepatic space. The drain tube was removed on the fourth post operative day. The post-operative period was uneventful. On the eighth post-operative day the child was discharged on triple H-Pylori therapy. ${ }^{2}$ After a follow up of three weeks the patient had recovered completely.

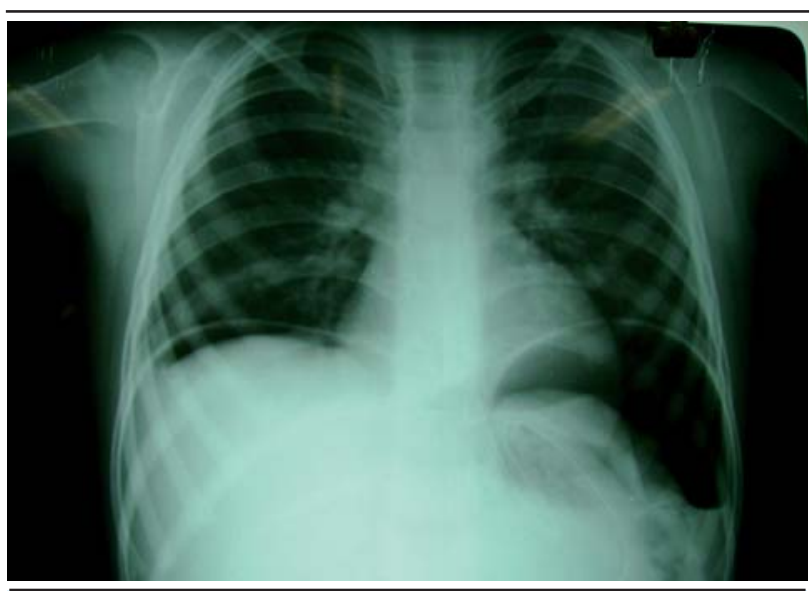

Figure 1. Chest X-ray showing free gas under diaphragm (white arrow)

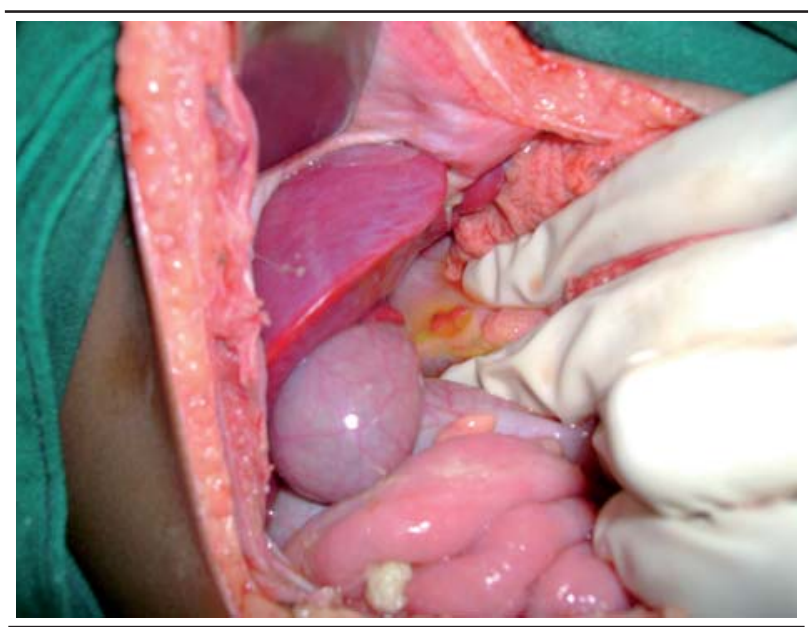

Figure 2. Operative photograph showing a small perforation in the anterior wall of the first part of duodenum (black arrow)

\section{DISCUSSION}

Duodenal ulcer is usually overlooked because of vague and variable symptoms and low index of suspicion on part of the treating physicians. ${ }^{3}$ With the increase use of endoscopy, it has been shown that peptic ulcer disease is not as uncommon as previously assumed. Over the past several years there has been three fold increase in the incidence of peptic ulcer disease in pediatric population. ${ }^{4}$ The overall incidence varies from region to region, but a large body of evidence indicates that it is probably under reported condition, since its diagnosis may be delayed or missed. Peptic ulcer in children can be primary associated with Zollinger Ellison syndrome, sickle cell anemia, H. pylori infection ${ }^{4}$, blood group $\mathrm{O}$, medications like non steroidal anti-inflammatory drugs (NSAID) and corticosteroids ${ }^{5}$ or physiological stress in burns, head injury, mucosal ischemia. Maudar et al implicated diet as a predisposing factor. ${ }^{1}$ Our patient had ' $\mathrm{O}$ ' blood group and no other known predisposing factors. Investigations to confirm $\mathrm{H}$. pylori infection is not done routinely. Usually the diagnosis of peptic ulcer in children is made due to complications like perforation and hemorrhage. ${ }^{6}$ Very few reports are available regarding the perforated duodenal ulcer in children. The diagnosis of perforated duodenal ulcer is often missed in the emergency setting due to low incidence and low index of suspicion. Children present late with shock $^{6}$ and carry high morbidity and mortality. During the past decade, several retrospective and prospective studies in adults have identified advanced age, female sex, delay in surgery of more than 12 hours, shock at admission, concomitant medical illness, surgical treatment by simple suture, and large perforation as risk factors related to mortality. ${ }^{7}$ Though the abdominal $X$ ray demonstrates pneumoperitoneum, the diagnosis of perforated duodenal ulcer is usually made on exploratory laparotomy for peritonitis. Since 1990, laparoscopic treatment for perforated peptic ulcer has been reported to be a safe option in many centers. ${ }^{8}$

The diagnosis of perforated duodenal ulcer in children is often missed in the emergency setting due to its low incidence and low index of suspicion. Children present late with shock and thus carry high morbidity and mortality. Though the abdominal radiograph demonstrates pneumoperitoneum, the diagnosis of perforated duodenal ulcer is usually made on exploratory laparotomy for peritonitis. 


\section{REFERENCES}

1. Maudar KK, Dutta J, Mitra S. Duodenal ulcer disease in children. World J Surg. 1980;4:261-5.

2. Malfertheiner $\mathrm{P}$, Megraud F, O'Morain C, Hungin AP, Jones R, Axon A, et al. Current concepts in the management of Helicobacter pylori infection--the Maastricht 2-2000 Consensus Report. Aliment Pharmacol Ther. 2002;16:167180.

3. Roma E, Kafritsa Y, Panayiotou J, Liakou R, Constantopoulos A. Is peptic ulcer a common cause of upper gastrointestinal symptoms? Eur J Pediatr. 2001;160:497-600.

4. Tam, PKH, Saing H, Lau TK. Diagnosis of peptic ulcer in children: the past and present. J Pediatric Surg. 1986;21:15-6.

5. Hernandez-Diaz S, Rodriguez AG. Association between nonsteroidal anti-inflammatory drugs and upper gastrointestinal tract bleeding perforation: An overview of epidemiologic studies published in 1990s. Arch Intern Med. 2000;160: 2093-99.

6. Moon D, Weeks D, Burgess B, O'Connor R. Perforated duodenal ulcer presenting with shock in a child. Am J Em Med. 1997;15:167-9.

7. Testini M, Portincasa P, Piccinni G, et al. Significant factors associated with fatal outcome in emergency open surgery for perforated peptic ulcer. World J Gastroenterol. 2003;9:233840.

8. Lee FY, Leung KL, Lai PB, et al. Selection of patients for laparoscopic repair of perforated peptic ulcer. Br J Surg. 2001;88:133-6. 